\title{
Tunable Superstructures of Dendronized Graphene Nanoribbons in Liquid Phase
}

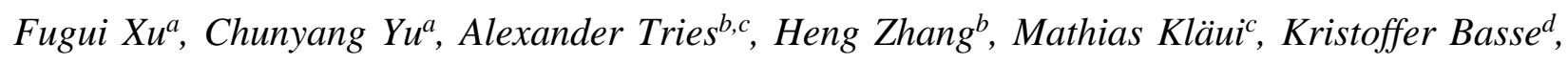
Michael Ryan Hansen ${ }^{e}$,Nerea Bilbaof, Steven De Feyter $f$, Mischa Bonn ${ }^{b}$, Hai I. Wang ${ }^{b}$, Yiyong $\mathrm{Mai}^{a *}$

a School of Chemistry and Chemical Engineering, Shanghai Key Laboratory of Electrical Insulation and Thermal Ageing, Shanghai Jiao Tong University, 800 Dongchuan Road, Shanghai 200240, China

${ }^{\mathrm{b}}$ Max Planck Institut für Polymerforschung, Ackermannweg 10, 55128 Mainz, Germany

c Graduate School Materials Science in Mainz, Staudingerweg 9, 55128 Mainz; Institut für Physik, Johannes Gutenberg-Universität, Staudinger Weg 7, 55128 Mainz, Germany

d Interdisciplinary Nanoscience Center, Aarhus University, Gustav Wieds Vej 14, DK-8000 Aarhus C, Denmark

e Institute of Physical Chemistry, Westfälische Wilhelms-Universität Münster, Corrensstr. 28/30, D-48149 Münster, Germany

$\mathrm{f}$ Division of Molecular Imaging and Photonics, Department of Chemistry, KU Leuven Celestijnenlaan, 200 F, B-3001 Leuven, Belgium

Abstract: Structurally well-defined graphene nanoribbons (GNRs) have attracted great interest due to their unique optical and electronic properties. Functionalization of GNRs with organic moieties may afford GNRs liquid-phase dispersibility and thus broaden their research scope. However, the candidates used for GNR functionalization have so far been limited to only a few small organic groups and poly(ethylene oxide) linear polymer, which impedes the development of new functional GNRs and their potential applications in liquid phase. Here, we report the first bottom-up synthesis of structurally well-defined GNRs functionalized with dendritic polymers. The resultant GNRs possess grafting ratios of 0.59-0.68 for the dendrons of different generations. Remarkably, the precise 3D branched configurations of the grafted dendrons afford the GNRs unprecedented 1D supramolecular self-assembly behavior in organic solvents such as tetrahydrofuran (THF), yielding nanowires, helices and nanofibers depending on the dimension of the dendrons. The GNR superstructures in THF exhibit near-infrared absorption with maxima between 650 and $700 \mathrm{~nm}$, yielding an optical bandgap of 1.2 1.3 eV. Ultrafast photoconductivity analyses unveil that the helical structures exhibit the longest free carrier (3.5 ps) and exciton lifetime (several hundred ps) among the three superstructure systems. This study opens avenues for tunable construction of ordered GNR superstructures with promising optoelectronic applications. 
Structurally well-defined graphene nanoribbons (GNRs) have attracted great interest due to their unique optical and electronic properties. ${ }^{1-17}$ For example, their optical and electronic bandgaps are governed by their width and edge structure, allowing promising applications in semiconductor devices, ${ }^{1,3,4,10}$ photothermal conversion, ${ }^{14}$ and super-resolution fluorescence imaging, ${ }^{15}$ among others. Structurally defined GNRs can be prepared by solution-mediated chemical synthesis, ${ }^{18-25}$ enabling the large-scale production of liquid-phase dispersible GNRs and their edge functionalization, in contrast to other synthetic strategies including top-down ${ }^{3,16,17}$ and surface-assisted bottom-up approaches. ${ }^{6,26-32}$ In recent years, significant effort has been devoted to the functionalization of GNRs with small organic molecules ${ }^{5,8,9,15,19,22-25}$ or polymers, ${ }^{12-14}$ endowing GNRs with good dispersibility and providing new opportunities for investigating their physiochemical properties in the liquid phase. The success of increasing the dispersibility and long-term stability of GNRs in liquid phase by grafting of polymer chains, rather than small organic molecules, can be traced to the larger geometric dimensions of polymers, which can more effectively alleviate the strong $\pi-\pi$ interaction of GNR backbones. ${ }^{13,14}$ However, polymerfunctionalization of GNRs has so far been limited to linear poly(ethylene oxide) (PEO) ${ }^{13}$, yet clearly additional functionalization could be introduced. This realization has inspired the interest to functionalize GNRs with other polymeric structures to achieve novel physiochemical properties and potential applications of the resultant GNRs.

Here, we report the solution synthesis of dendronized GNRs, which consist of a structurally defined backbone grafted with benzyl ether-type dendrons ${ }^{33-35}$ of different generations (GNR-G1, -G2 and -G3, Scheme 1). The GNR backbones possess an arm-chair edged structure with a uniform width of $1.7 \mathrm{~nm}$ and an average length of $30 \mathrm{~nm} \cdot{ }^{13}$ The side alkylcarboxyl active groups on the periphery of the backbones allow the grafting of benzyl ether-type dendrons (G1-G3) with 
hydroxyl groups through an esterification reaction. The resultant dendronized GNRs have grafting ratios (GRs) of $0.59-0.68$ for the dendrons of different generations. The bulky threedimensional (3D) dendrons, which may alleviate the $\pi-\pi$ stacking of GNR backbones, render the GNRs with excellent dispersibility in common solvents such as tetrahydrofuran (THF). The maximum concentration of the GNR dispersion in THF reaches $\sim 3 \mathrm{mg} \mathrm{mL}^{-1}$ (for the GNR backbone unless otherwise mentioned), which significantly exceeds those of reported alkyl chainmodified $\left(0.01 \mathrm{mg} \mathrm{mL}^{-1}\right)^{5}$ or PEO-functionalized GNRs $\left(1 \mathrm{mg} \mathrm{mL}^{-1}\right)^{13}$. More interestingly, in THF dispersions $\left(0.01 \sim 1 \mathrm{mg} \mathrm{mL}^{-1}\right)$ the dendronized GNRs aggregate into ultralong nanowires, 1D helices, or short nanofibers, depending on the dimension of the dendrons, which governs the aggregation mode of the GNRs. Ordered GNR superstructures have been rarely achieved thus far due to the limited dispersibility of GNRs. The present 1D nanostructures, to our knowledge, represent the first ordered GNR superstructures in organic solvents, thanks to the well-defined geometry of the grafted dendrons. ${ }^{33-35}$ The formation of the superstructures leads to near-infrared (NIR) absorption with an absorption maximum at $685 \mathrm{~nm}$ for GNR-G1 and GNR-G2 in THF, and at $652 \mathrm{~nm}$ for GNR-G3. Ultrafast photoconductivity measurements unveil the charge carrier dynamics in the three GNR superstructure systems. Excitons are found to be long-lived with lifetimes of several hundred picoseconds. Remarkably, the GNR-G2 helices exhibit a much longer free carrier (3.5 ps) and exciton lifetime (several hundred ps) than those of GNR-G1 and G3 with nanowire and short nanofiber superstructures, as well as those of reported GNRs ${ }^{5,38}$. This discrepancy indicates the considerable effect of supramolecular structures on the optoelectronic properties of GNRs in the liquid phase, making them promising candidates for optoelectronics applications. 
The G1-G3 dendrons were synthesized by a traditional convergent method ${ }^{33,35}$. The production of the dendrons was demonstrated by ${ }^{1} \mathrm{H},{ }^{13} \mathrm{C}$ NMR and matrix-assisted laser desorption/ionization time-of-flight (MALDI-TOF) mass spectra (see details in the Supporting Information, SI). The dendronized GNRs were synthesized by the esterification of the hydroxyl groups in the dendrons with the carboxyl groups at the edges of structurally defined GNRs (GNR-COOH ${ }^{13}$, Scheme 1). The Fourier transform infrared (FTIR) spectra of GNR-G1-3 (Figure $\mathrm{S} 1)$ show a distinct increase in the intensity of the signal at $1730 \mathrm{~cm}^{-1}(\mathrm{C}=\mathrm{O}$ stretching from the ester group) and an attenuation of the band at $1702 \mathrm{~cm}^{-1}(\mathrm{C}=\mathrm{O}$ stretching from the carboxyl group), demonstrating the successful grafting of the dendrons. ${ }^{12,13} 2 \mathrm{D}$ solid-state ${ }^{1} \mathrm{H}-{ }^{1} \mathrm{H}$ double quantum-single quantum (DQ-SQ) MAS NMR spectra confirm the dendron grafting and the unaffected GNR backbone after the grafting (Figure S2). ${ }^{13,22}$ Quantitative single-pulse solid-state ${ }^{13} \mathrm{C}$ magic-angle spinning (MAS) NMR spectra give dendron grafting ratios of $0.59,0.60$, and 0.68 for GNR-G1, -G2 and -G3, respectively (Figure S3, Table S1). The first-order Raman spectra of the dendronized GNRs exhibit typical D and G peaks (Figure S4), which are basically identical to those of GNR-COOH, ${ }^{13}$ indicating that the dendron grafting does not affect the graphitic structure of the GNR backbones.

Mild sonication of the dendronized GNRs in common organic solvents, such as THF, chloroform, toluene, chlorobenzene, etc., generated blue-black homogeneous dispersions, which were stable without visible precipitation for over one day depending on the generation of the dendrons (Figure 2a). As the generation of the grafted dendron increases, GNR-G1, -G2 and -G3 exhibit improved dispersibility, e.g. in THF, with the highest concentrations of $1.5,2.4$, and $3 \mathrm{mg}$ $\mathrm{mL}^{-1}$, respectively. The maximum concentration of the GNR-G3 dispersion is three times higher than that $\left(\sim 1 \mathrm{mg} \mathrm{mL}^{-1}\right)$ of PEO-grafted GNRs ${ }^{13}$ and two orders of magnitude higher than those $\left(\sim 0.01 \mathrm{mg} \mathrm{mL}^{-1}\right)$ of the reported alkylated GNRs, highlighting the much better effect of the 3D 
dendrons on alleviating the strong $\pi-\pi$ stacking of GNRs. GNR-G3 in dilute THF dispersion $\left(0.01 \mathrm{mg} \mathrm{mL}^{-1}\right)$ shows optical absorption between 500 and $1200 \mathrm{~nm}$ with the maximum at $\sim 652$ $\mathrm{nm}$, which yields an optical bandgap of $\sim 1.3 \mathrm{eV}$ (Figure $2 \mathrm{~b}$ ). In contrast, GNR-G1 and -G2 in THF dispersions show red-shifted absorptions in NIR region with the maximum at $\sim 685 \mathrm{~nm}$, suggesting increased aggregation compared with GNR-G3 under similar conditions.

The differences in UV-vis-NIR absorptions suggest electronic coupling between GNRs in the dispersions, mediated by their functionalization. This implies that defined aggregates exist in THF dispersions, which were examined by transmission electron microscopy (TEM), cryo-TEM and atomic force microscopy (AFM). Interestingly, GNR-G1, -G2 and -G3 formed different superstructures, including ultralong nanowires, 1D helices, and short nanofibers (Figure 3). The morphologies of these aggregates were nearly independent of the GNR concentrations in the range of $0.01 \sim 1 \mathrm{mg} \mathrm{mL}$. The resultant superstructures were stable without obvious morphological changes during the time span of the experiments. As a control, ordered superstructures have never been documented for GNRs grafted with alkyl or PEO chains in organic solvents, emphasizing the exceptional effect of the dendrons on the aggregation mode of GNRs.

GNR-G1 formed ultralong wire-like nanostructures with a mean diameter of $40 \pm 25 \mathrm{~nm}$ and lengths of $15-50 \mu \mathrm{m}$ based on the analysis of 200 objects in TEM images (Figure 3a,b, Figure S5). AFM height profiles confirm the formation of the nanowires and give an average diameter of 45 $\pm 28 \mathrm{~nm}$ (Figure 3c), which is larger than that obtained by TEM. Considering the sizes of the nanowires and the GNRs as well as the synergistic effect of the $\pi-\pi$ interaction of the GNRs and the tight packing of the grafted G1 dendron with symmetric configuration, a possible aggregation model for the nanowires is proposed in Figure $3 \mathrm{~d}$. The strong $\pi$ - $\pi$ interaction of GNRs resulted in 
the aggregation of GNR-G1, in an entropically-driven random side-by-side and end-to-end fashion. The association led to a crowded packing of the G1 groups on the periphery of the aggregated ribbons, which could induce the transformation of G1 to a more energetically favorable near-fan architecture (see calculation in Page S15). ${ }^{33,34}$ The tight arrangement of the fan-shaped dendrons limited the aggregation of GNR-G1 preferentially along the 1D direction, yielding the nanowires (Figure 3d). The aggregation model may also account for the smaller average diameter measured by TEM than that by AFM, due to the coverage of the dendrons that are low-contrast and generally invisible under TEM observation. ${ }^{34,35}$

More interestingly, GNR-G2 aggregated into ultralong helical nanostructures (Figure 3e-g, Figure S6); their dimensions are given in the related TEM or AFM images. The formation of the helices in the liquid phase was confirmed by cryo-TEM (inset of Figure 3e). The coexistence of left- and right-handed helices was observed. Most of the helices are double- or triple-stranded, which consist of the twist of thinner wire-like nanostructures. Similar to the GNR-G1 nanowires, the formation of the helices can also be driven by the interplay between the GNR $\pi$ - $\pi$ stacking and the tight arrangement of the G2 dendrons (Figure 3h). However, the difference is the larger dimension of the dendrons. It is known that the formation of racemic helical structures by the self-assembly of a few achiral block copolymer systems could be driven by steric hindrance among polymer chains. ${ }^{36,37}$ Thereby, we consider that the formation of the GNR helices is driven by the steric hindrance among the G2 dendrons. For GNR-G2, the average distance between the adjacent G2 dendrons on the GNR backbone $(d \sim 1.1 \mathrm{~nm})$ is much smaller than twice the estimated radius $\left(2 R_{\mathrm{G} 2} \sim 3.2 \mathrm{~nm}\right)$ of a $\mathrm{G} 2$ dendron (see calculation in Page S15). After the aggregation of the GNRs, the steric hindrance between the neighboring G2 dendrons is quite large, which results in a more energetically favorable near-fan configuration of $\mathrm{G} 2,{ }^{33,34}$ and a 
spiral of the associated semirigid GNRs, ${ }^{3,5}$ providing more peripheral space for accommodation of the dendrons (Figure 3h). Meanwhile, the contribution of entropy leads to the simultaneous formation of the left- and right-handed helices. In contrast, the average distance between the neighboring G1 dendrons on the GNR backbone $(d \sim 1.1 \mathrm{~nm})$ is only slightly smaller than twice the radius $\left(2 R_{\mathrm{G} 1} \sim 1.5 \mathrm{~nm}\right)$ of a $\mathrm{G} 1$ dendron (Page S15). The resulting steric hindrance can drive the transformation of G1, but is insufficient to induce a spiral of the associated GNRs.

Under similar conditions, GNR-G3 formed short nanofibers with average diameter and length of $10 \pm 3 \mathrm{~nm}$ and $120 \pm 45 \mathrm{~nm}$, respectively (Figure 3i,j, Figure S7). The formation mechanism resembles that of the GNR-G1 nanowires (Figure 3k), but the much greater dimension of G3 significantly reduces the number of aggregation $\left(N_{\mathrm{agg}}\right)$, leading to an apparent decrease in the length and diameter of the nanofibers. This reduced tendency for aggregation may account for the fact that it was hard to find the short and thin fibers under TEM, due to their insufficient contrast.

To testify the possibility for the formation of the above-discussed 1D superstructures, dissipative particle dynamics (DPD) simulations were performed (see details in Pages S16-S19). The GNR-dendron molecules are modeled as coarse-grained brush-like bead-spring chains, $\mathrm{A}_{\mathrm{x}} \mathrm{B}_{\mathrm{y}}$, with $\mathrm{x}$ A-beads in the GNR backbone and y B-beads denoting the dendron segments. Three model molecules including $\mathrm{A}_{69} \mathrm{~B}_{23}, \mathrm{~A}_{69} \mathrm{~B}_{92}$, and $\mathrm{A}_{69} \mathrm{~B}_{299}$ are constructed to represent GNR-G1, G2, and -G3, respectively (Figure 4a). Figure 4b displays snapshots during the formation of GNR-G1 nanowires, in which the $\mathrm{A}_{69} \mathrm{~B}_{23}$ molecules (Figure $4 \mathrm{~b} 1$ ) self-assemble into numerous thin fibers (Figure 4b2), and the latter gradually associate into long nanowires with a combined side-by-side and end-to-end alignment of "GNR backbones" (Figure $4 \mathrm{~b} 3$ and the inset). For $\mathrm{A}_{69} \mathrm{~B}_{92}$ (GNR-G2) molecules, small short helices and irregular aggregates form firstly (Figure $4 c 2$ ) after the initial random state (Figure $4 \mathrm{c} 1$ ); these small aggregates gradually evolve into long helices, in which the associated GNR backbones are twisted upon the repulsion of the dendrons 
(Figure 4c3 and the inset). With the largest dendrons, most $\mathrm{A}_{69} \mathrm{~B}_{299}$ (GNR-G3) molecules aggregate into short nanofibers with a GNR packing mode similar to that in the $\mathrm{A}_{69} \mathrm{~B}_{23}$ nanowires, while a minority of $\mathrm{A}_{69} \mathrm{~B}_{299}$ retain their unimolecular state (Figure 4d3). Evidently, the DPD simulations nicely support the experimental results and the packing modes of the GNRs in the superstructurs proposed in Figure 3.

To understand the role of dendron-functionalization and superstructures on the optoelectronic properties of GNRs, we evaluate the ultrafast photoconductivity of GNR-G1, -G2, and -G3 dispersed in THF employing time-resolved optical pump-Terahertz (THz) probe (OPTP) spectroscopy. ${ }^{38-43}$ The measurement protocol is shown in Figure S10 and the underlying principle is discussed in the SI (Pages S19-S20). Figure 5 compares the time-dependent complex conductivity of GNR G1-G3 in THF dispersions $(\sim 1 \mathrm{mg} / \mathrm{mL})$. In principle, the real part of the conductivity provides the information of the free carrier conductivity, while the imaginary part reflects the change in refractive index due to the photoexcitation. ${ }^{38,44}$ In the absence of free charges, the imaginary part reflects the polarizability of excitons. ${ }^{45}$ The peak in the real conductivity has been normalized for comparison, and the imaginary part is rescaled accordingly. Obviously, the $\mathrm{THz}$ response rises sharply upon photoexcitation, followed by a rapid decay in both the real and the imaginary parts. After the initial fast decay, the imaginary conductivity (the signature of excitons) remains substantial, in contrast to the real conductivity (the signature of free carriers), which goes to zero. In line with previous reports $5,38,39,45,46$, the ultrafast rise of the real conductivity signal is attributed to the generation of short-lived quasi-free charge carriers; the subsequent rapid decay arises from the formation of excitons within a few ps due to strong Coulomb interactions between photogenerated electron-hole pairs. The exciton states are longlived, several hundreds of picoseconds (Figure S11). While the photoconductivity dynamics of all three different GNRs qualitatively reflects charge generation followed by exciton formation, 
quantitative differences are observed between GNR-G1 and -G3 on the one hand, and GNR-G2 on the other. The photoconductivity of GNR-G1 and -G3 with similar 1D superstructure (nanowires and nanofibers, respectively), is characterized by a nearly-identical 0.7 ps decay time constant, in spite of their striking variance in dendron size. In contrast, GNR-G2 helical superstructures exhibit a five-fold longer free carrier lifetime (3.5 ps) than those of GNR-G1 and -G3, which is also longer than those of reported alkyl-chain modified GNRs ${ }^{5,38}$. These results indicate that the measured photoconductivity of GNRs does not originate from single nanoribbons, as otherwise the free carrier and exciton dynamics among all ribbons would be identical. Instead, our results illustrate the vital effect of GNR superstructures on the lifetime of free charge and exciton formation dynamics. The relatively long-lived free charge carriers in GNR-G2 helical structures make them a promising candidate for optoelectronics applications as it may offer enhanced free charge carrier escape and extraction before the formation of excitons.

In summary, we demonstrate the solution synthesis of dendronized GNRs, which consist of a structurally defined GNR backbone grafted with benzyl ether-type dendrons. The 3D dendrons render the GNRs with superior dispersibility and tunable 1D superstructures including nanowires, helices and nanofibers, depending on the dimension of the dendrons. DPD simulations reveal that the unprecedented self-assembly behavior is attributable to the synergistic effect of the $\pi-\pi$ interaction of the GNRs and the tight packing of the grafted dendrons with symmetric geometry. The charge carrier dynamics in the three superstructure systems was studied by ultrafast photoconductivity measurements, unveiling free carrier lifetime of 3.5 ps and exciton lifetime of several hundred ps for the helices, much longer than those of the other two superstructures. Dendronized GNRs thus hold promise as some of powerful building units for the construction of 
1D functional nanomaterials with potential applications in optoelectronics, nanocomposites, biotechnology, among others.

\section{ASSOCIATED CONTENT}

\section{Supporting Information}

Experiments details, supporting figures and calculations, etc.

\section{AUTHOR INFORMATION}

\section{Corresponding Authors}

*mai@sjtu.edu.cn (Y. M.)

\section{Notes}

The authors declare no competing financial interest.

\section{ACKNOWLEDGMENTS}

The authors thank Prof. Klaus Müllen at MPIP and Prof. Xinliang Feng at Technische Universität Dresden for kind suggestions. The authors also appreciate the financial support from National Natural Science Foundation of China (21774076 and 51573091), Program of the Shanghai Committee of Science and Technology (17JC1403200), Program of Shanghai Academic Research Leader (19XD1421700) and Program of Shanghai Eastern Scholar. In Mainz the work was supported by the DFG (in particular GSC/266 Graduate School of Excellence Materials Science in Mainz). 


\section{REFERENCES}

1. Y. W. Son, M. L. Cohen, S. G. Louie, Nature 2006, 444, 347-349.

2. $\quad$ K. A. Ritter, J.W. Lyding, Nat. Mater. 2009, 8, 235-242.

3. X. Li, X. Wang, L. Zhang, H. Dai, Science 2008, 319, 1229-1232.

4. A. H. C. Neto, F. Guinea, N. M. R. Peres, K. S. Novoselov, A. K. Geim, Rev. Mod. Phys. 2009, 81, $109-164$.

5. A. Narita, X. Feng, Y. Hernandez, S. A. Jensen, M. Bonn, H. Yang, I. A. Verzhbitskiy, C. Casiraghi, M. R. Hansen, A. H. R. Koch, G. Fytas, O. Ivasenko, B. Li, K. S. Mali, T. Balandina, S. Mahesh, S. D. Feyter, K. Müllen, Nat. Chem. 2014, 6, 126-132.

6. P. Ruffieux, S. Wang, B. Yang, C. Sánchez-Sánchez, J. Liu, T. Dienel, L. Talirz, P. Shinde, C. A. Pignedoli, D. Passerone, T. Dumslaff, X. Feng, K. Müllen, R. Fasel, Nature 2016, 531, 489-492.

7. R. S. Jordan, Y. L. Li, C.W. Lin, R. D. McCurdy, J. B. Lin, J. L. Brosmer, K. L. Marsh, S. I. Khan, K. N. Houk, R. B. Kaner, Y. Rubin, J. Am. Chem. Soc. 2017, 139, 15878-15890.

8. T. J. Sisto, Y. Zhong, B. Zhang,M. T. Trinh, K. Miyata, X. Zhong, X. Y. Zhu, M. L. Steigerwald, F. Ng, C. Nuckolls, J. Am. Chem. Soc. 2017, 139, 5648-5651.

9. E. Castro, T. J. Sisto, E. L. Romero, F. Liu, S. R. Peurifoy, J. Wang, X.Y. Zhu, C. Nuckolls, L. Echegoyen, Angew. Chem. Int. Ed. 2017, 56, 14648-14652.

10. M. Han, B. zyilmaz, Y. Zhang, P. Kim, Phys. Rev. Lett. 2007, 98, 206805.

11. M. Slota, A. Keerthi, W. K. Myers, E. Tretyakov, M. Baumgarten, A. Ardavan, H. Sadeghi, C. J. Lambert, A. Narita, K. Müllen, L. Bogani, Nature 2018, 557, 691-695.

12. Y. Huang, Y. Mai, X. Yang, U. Beser, J. Liu, F. Zhang, D. Yan, K. Müllen, X. Feng, J. Am. Chem. Soc. 2015, 137, 11602-11605.

13. Y. Huang, Y. Mai, U. Beser, J. Teyssandier, G. Velpula, H. Gorp, L. A. Hansen, M. R. Hansen, D. Rizzo, C. Casiraghi, R. Yang, G. Zhang, D. Wu, F. Zhang, D. Yan, S. D. Feyter, K. Müllen, X. Feng, J. Am. Chem. Soc. 2016, 138, 10136-10139.

14. Y. Huang, W. Dou, F. Xu, H. Ru, Q. Gong, D. Wu, D. Yan, H. Tian, X. He, Y. Mai, X. Feng, Angew. Chem. Int. Ed. 2018, 57, 3366-3371.

15. D. Joshi, M. Hauser, G. Veber, A. Berl, K. Xu, F. R. Fischer, J. Am. Chem. Soc. 2018, 140, 9574-9580.

16. D. V. Kosynkin, A. L. Higginbotham, A. Sinitskii, J. R. Lomeda, A. Dimiev, B. K. Price, J. M. Tour, Nature 2009, 458, 872-876.

17. X. Wang, H. Dai, Nat. Chem. 2010, 2, 661-665.

18. M. Schwab, A. Narita, Y. Hernandez, T. Balandina, K. S. Mali, S. De Feyter, X. Feng, K. Müllen, J. Am. Chem. Soc. 2012, 134,18169-18172.

19. M. Daigle, D. Miao, A. Lucotti, M. Tommasini, J.F. Morin, Angew. Chem. Int. Ed. 2017, 56, 6213- 
6217.

20. W. Yang, A. Lucotti, M. Tommasini, W. A. Chalifoux, J. Am.Chem. Soc. 2016, 138, 9137-9144.

21. L. Dssel, L.Gherghel, X. L. Feng, K. Müllen, Angew. Chem. Int. Ed. 2011, 50, 2540-2543.

22. Y. Huang, F. Xu, L. Ganzer, F. V. A. Camargo, T. Nagahara, J. Teyssandier, H. V. Gorp, K. Basse, L. A.Straasø, V. Nagyte, C. Casiraghi, M. R. Hansen, S. D. Feyter, D. Yan, K. Müllen, X. Feng, G. Cerullo, Y. Mai, J. Am. Chem. Soc. 2018, 140, 10416-10420.

23. D. Miao, M. Daigle, A. Lucotti, J. Boismenu-Lavoie, M. Tommasini, J. F. Morin, Angew. Chem. Int. Ed. 2018, 57, 3588-3592.

24. G. Li, K.Y. Yoon, X. Zhong, J. Wang, R. Zhang, J. R. Guest, J. Wen, X. Y. Zhu, G. Dong, Nat Commun. 2018, 9, 1687-1696.

25. Y. Hu, P. Xie, M. D. Corato, A. Ruini, S. Zhao, F. Meggendorfer, L.A. Straas $\varnothing$, L. Rondin, P. Simon, J. Li, J. J. Finley, M. R. Hansen, J. S. Lauret, E. Molinari, X. Feng, J. V. Barth, C. A. Palma, D. Prezzi, K. Müllen, A. Narita, J. Am. Chem. Soc. 2018, 140, 7803-7809.

26. J. Cai, P. Ruffieux, R. Jaafar, M. Bieri, T. Braun, S. Blankenburg, M. Muoth, A. P. Seitsonen, M. Saleh, X. Feng, K. Müllen, R. Fasel, Nature 2010, 466, 470-473.

27. Y. C. Chen, T. Cao, C. Chen, Z. Pedramrazi, D. Haberer, D. G. Oteyza, F. R. Fischer, S. G. Louie, M. F. Crommie, Nat. Nanotechnol. 2015, 10, 156-160.

28. S. Wang, L. Talirz, C. A. Pignedoli, X. Feng, K. Müllen, R. Fasel, P. Ruffieux, Nat. Commun. 2016, 7, 11507-11511.

29. C. Ma, Z. Xiao, H. Zhang, L. Liang, J. Huang, W. Lu, B. G. Sumpter, K. Hong, J. Bernholc, A. P. Li, Nat. Commun. 2017, 8, 14815-14822.

30. H. Sakaguchi, S. Song, T. Kojima, T. Nakae, Nat. Chem. 2017, 9, 57-63.

31. R. A. Durr, D. Haberer, Y. Lee, R. Blackwell, A. M. Kalayjian, T. Marangoni, J. Ihm, S. G. Louie, F. R. Fischer, J. Am. Chem. Soc. 2018, 140, 807-813.

32. Z. Pedramrazi, C. Chen, F. Zhao, T.Cao, G. D. Nguyen, A. A. Omrani, H. Tsai, R. R. Cloke, T. Marangoni, D. J. Rizzo, T. Joshi, C. Bronner, W. Choi, F.R. Fischer, S. G. Louie, M. F. Crommie, Nano Lett. 2018, 18, 3550-3556.

33. V. S. K. Balagurusamy, G. Ungar, V. Percec, G. Johansson, J. Am. Chem. Soc. 1997, 119, 1539-1555.

34. K. V. Rao, D. Miyajima, A. Nihonyanagi, T. Aida, Nat. Chem. 2017, 9, 1133-1139.

35. A. D. Schlüter, J. P. Rabe, Angew. Chem. Int. Ed. 2000, 39, 864-883.

36. (a) Zhang, S.; Cui, H.; Chen, Z.; Wooley, K. L.; Pochan, D. J. Soft Matter 2008, 4, 90-98; (b) J. Dupont, G. J. Liu, K. Niihara, R. Kimoto, H. Jinnai, Angew. Chem. Int. Ed. 2009, 48, 6144-6147;

37. J. Bae, J. Choi, Y. Yoo, N. Oh, B. Kim, M. Lee, J. Am. Chem. Soc. 2005, 127, 9668-9669.

38. I. Ivanov, Y. B. Hu, S. Osella, U. Beser, H. I. Wang, D. Beljonne, A. Narita, K. Müllen, D. 
Turchinovich, M. Bonn, J. Am. Chem. Soc., 2017, 139, 7982-7988.

39. Z. P. Chen, H. I. Wang, J. Teyssandier, K. S. Mali, T. Dumslaff, I. Ivanov, W. Zhang, P. Ruffieux, R. Fasel, H. J. Räder, D. Turchinovich, S. D. Feyter, X. Feng, M. Kläui, A. Narita, M. Bonn, K. Müllen, J. Am. Chem. Soc., 2017, 139, 3635-3638.

40. Z. P. Chen, H. I. Wang, N. Bilbao, J.Teyssandier, T. Prechtl, N. Cavani, A. Tries, R. Biagi, V. D. Renzi, X. Feng, M. Kläui, S. D. Feyter, M. Bonn, A. Narita, K. Müllen, J. Am. Chem. Soc., 2017, 139, 94839486.

41. S. A. Jensen, R. Ulbricht, A. Narita, X. Feng, K. Müllen, T. Hertel, D. Turchinovich, M Bonn, Nano Lett., 2013, 13, 5925-5930.

42. A. Narita, I. A. Verzhbitskiy, W. Frederickx, K. S. Mali, S. A. Jensen, M. R. Hansen, M. Bonn, S. D. Feyter, C. Casiraghi, X. Feng, K. Müllen, ACS Nano, 2014, 8,11622-11630.

43. Z. Y. Liu, H. I. Wang, A. Narita, Q. Chen, Z. Mics, D. Turchinovich, M. Kläui, M. Bonn, K. Müllen, J. Am. Chem. Soc., 2017, 139, 9443-9446.

44. R. Ulbricht, E. Hendry, J. Shan, T. F. Heinz, M. Bonn, Rev. Mod. Phys. 2011, 83, 543.

45. F. Wang, J. Shan, M. A. Islam, I. P. Herman, M. Bonn, T. F. Heinz, Nat. Mater. 2006, 5, 861-864.

46. E. Hendry, J. M. Schins, L. P. Candeias, L. D. A. Siebbeles, M. Bonn, Phys. Rev. Lett. 2004, 92, 196601. 


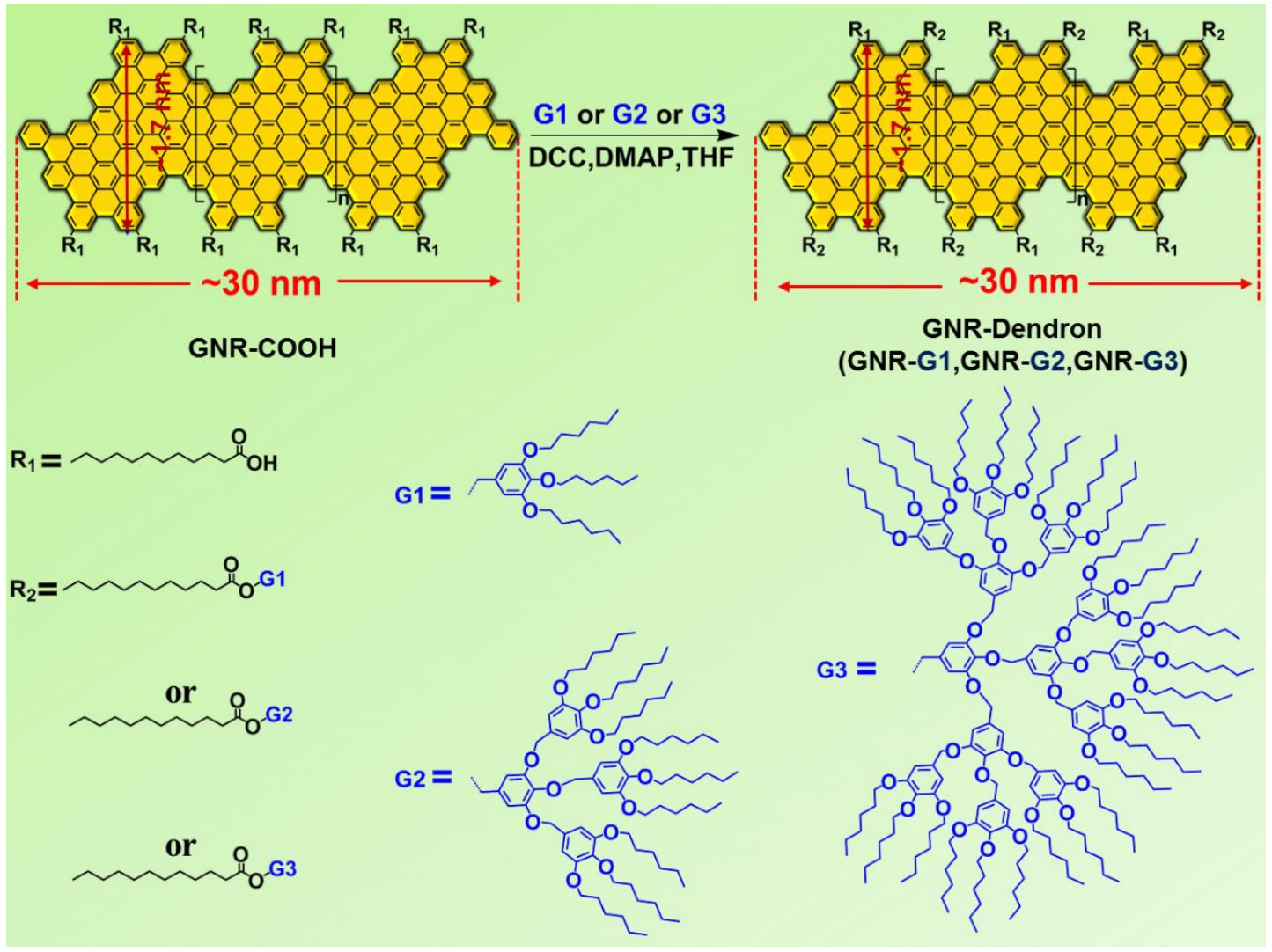

Figure 1. Schematic illustration of the synthesis of the dendronized GNRs (GNR-G1, -G2 and G3). 

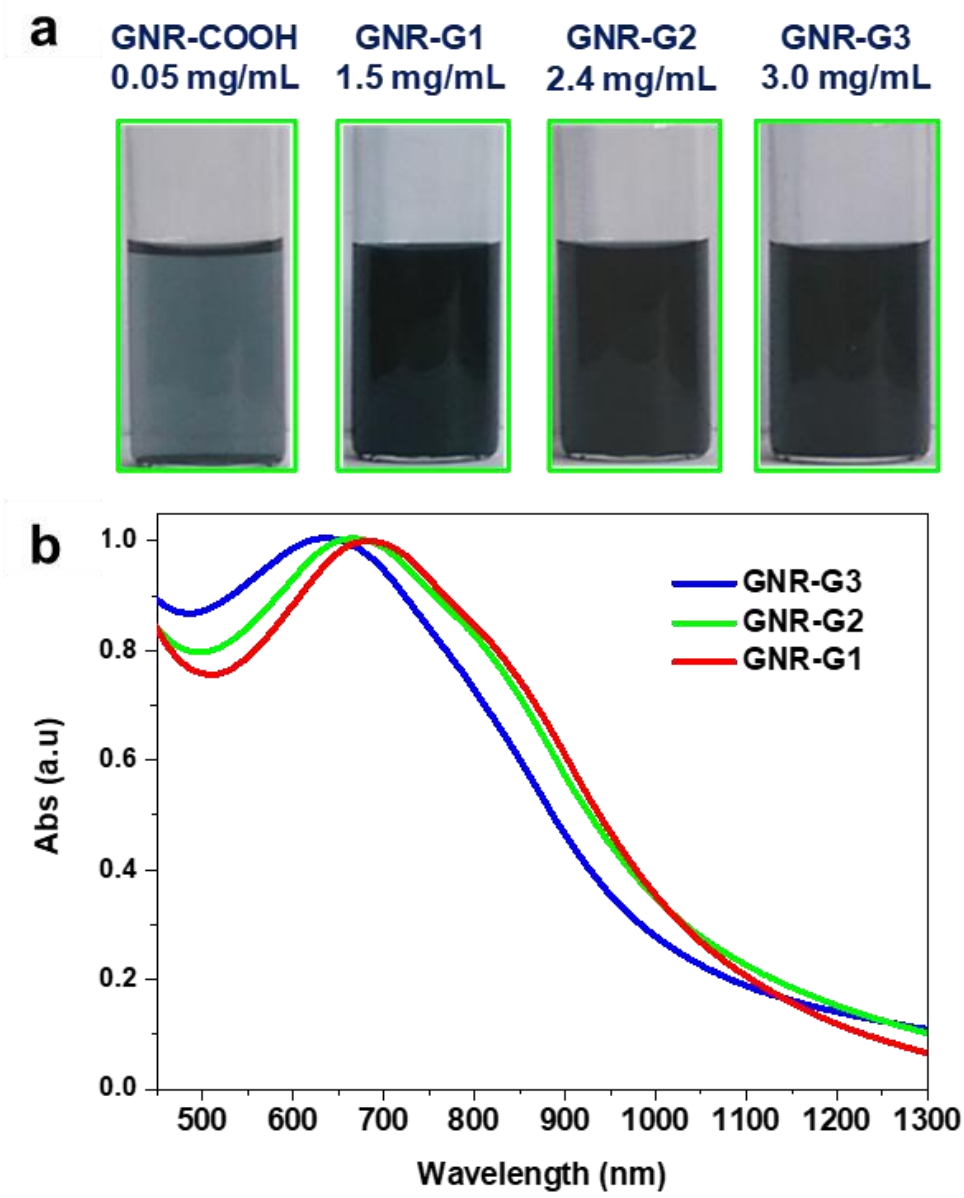

Figure 2. (a) Dispersion of GNR-COOH and the dendronized GNRs in THF with the maximum concentrations. (b) The normalized UV-vis absorption spectra of the dendronized GNRs in THF (conc.: $0.01 \mathrm{mg} \mathrm{mL}^{-1}$ ). 


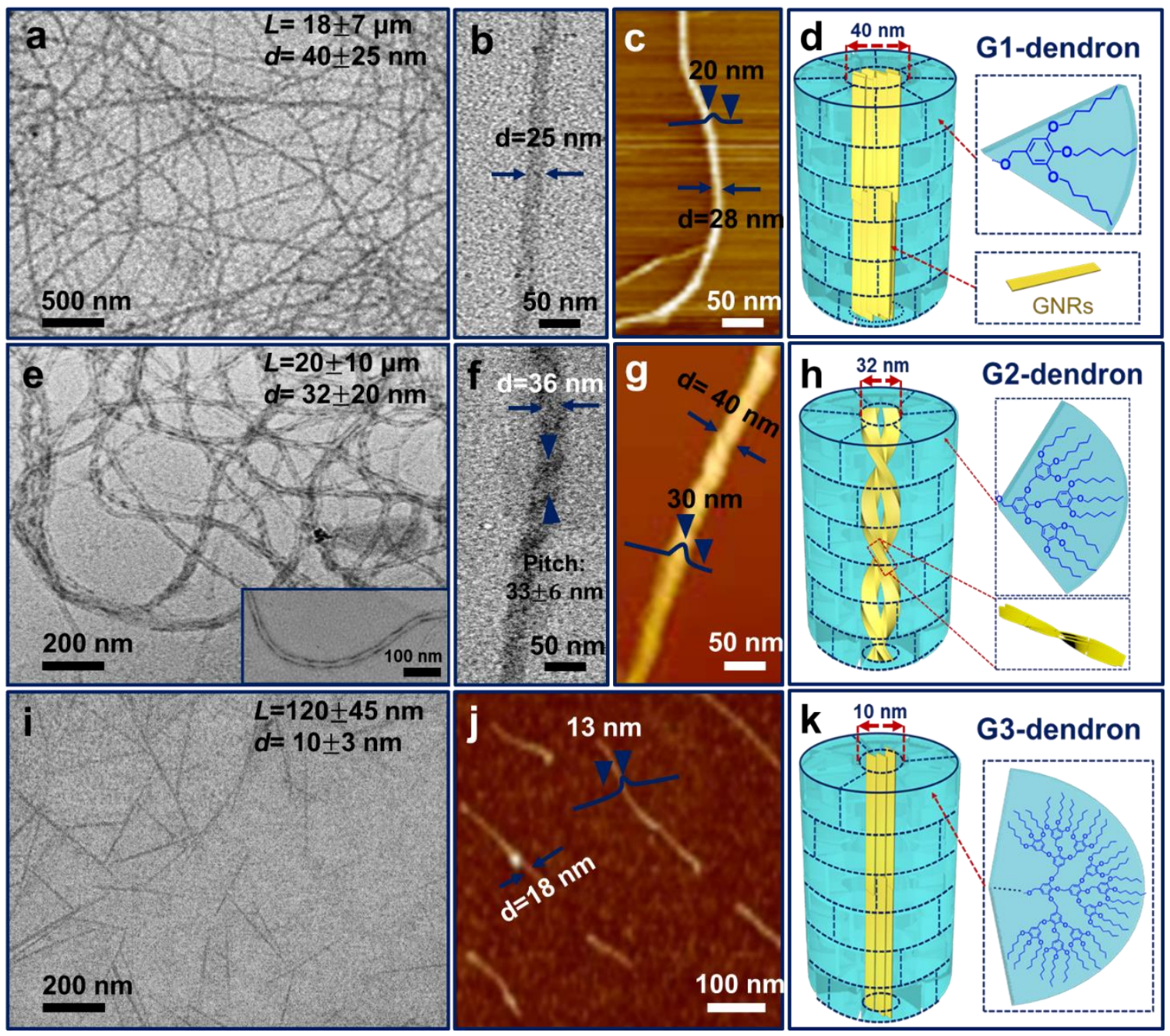

Figure 3. TEM images, AFM height profiles and the proposed aggregation models of the superstructures formed by the dendronized GNRs in THF. (a-d) GNR-G1 nanowires; (e-h) GNRG2 helices, the inset in (e) shows a typical cryo-TEM image; (i-k) GNR-G3 nanofibers. Note that the schematic aggregation models $(\mathrm{d}, \mathrm{h}, \mathrm{k})$ are not drawn according to the actual sizes. For convenient illustration, the short alkyl chains between the GNR backbones and dendrons are omitted; the near-fan architectured dendrons are presented as "regular fans" separated by dashed lines, which do not represent the real "borders" among the dendrons. 


\section{(a)}
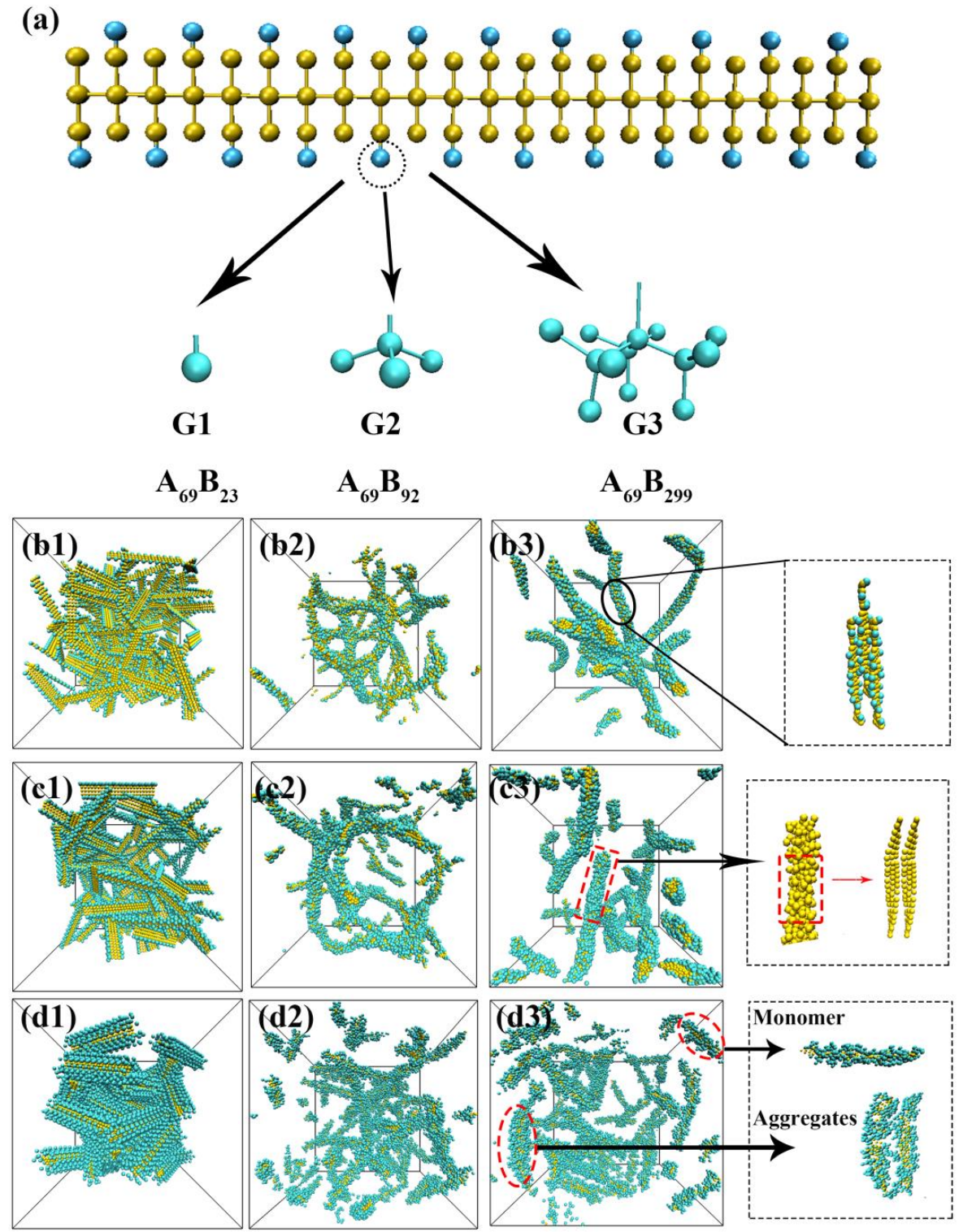

Figure 4. DPD simulation for the formation of the GNR-G superstructures in THF. (a) $\mathrm{A}_{69} \mathrm{~B}_{23}$, $\mathrm{A}_{69} \mathrm{~B}_{92}$ and $\mathrm{A}_{69} \mathrm{~B}_{299}$ represent GNR-G1, GNR-G2 and GNR-G3, respectively. (b1-b3) The formation process of $\mathrm{A}_{69} \mathrm{~B}_{23}$ nanowires at different simulation time: (b1) $\mathrm{T}=0$; (b2) $\mathrm{T}=1 \times 10^{4}$; (b3) $\mathrm{T}=2 \times 10^{6}$; the inset shows the packing model of $\mathrm{A}_{69} \mathrm{~B}_{23}$. (c1-c3) The formation process of $\mathrm{A}_{69} \mathrm{~B}_{92}$ helices at different time: (c1) $\mathrm{T}=0$; (c2) $\mathrm{T}=2 \times 10^{4}$; (c3) $\mathrm{T}=2.0 \times 10^{6}$; the inset shows the packing model of $\mathrm{A}_{69} \mathrm{~B}_{92} .(\mathrm{d} 1-\mathrm{d} 3)$ The formation process of $\mathrm{A}_{69} \mathrm{~B}_{299}$ nanofibers at different time: (c1) $\mathrm{T}=0$; (c2) $\mathrm{T}=5 \times 10^{5}$; (c4) $\mathrm{T}=2.0 \times 10^{6}$. Yellow: GNR backbone, cyan: dendrons. 


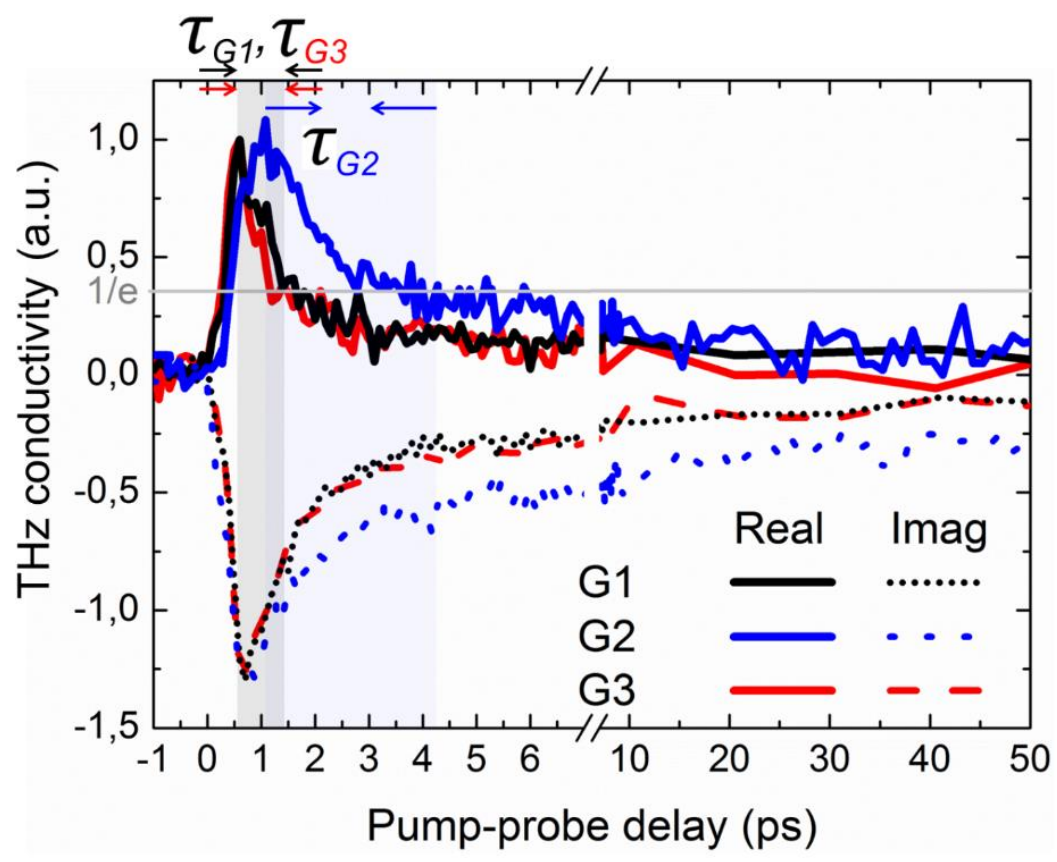

Figure 5. THz spectroscopy for monitoring the photoconductivity in GNRs. The plot summarizes and compares the time-resolved $\mathrm{THz}$ photoconductivity for GNR-G1, -G2, and -G3. The lifetimes $(\tau)$ of free charge carriers are defined by the decay time from the peak to $1 / \mathrm{e}$ in the real conductivity, as indicated by the marked shadow areas for GNR-G1-G3 dynamics. 